\title{
Mesenchymal stem cells derived from human induced pluripotent stem cells retain adequate osteogenicity and chondrogenicity but less adipogenicity
}

Ran Kang ${ }^{1,2+}$, Yan Zhou ${ }^{3+}$, Shuang Tan ${ }^{3,4}$, Guangqian Zhou ${ }^{4 *}$, Lars Aagaard ${ }^{3}$, Lin Xie² Cody Bünger $^{1}$, Lars Bolund ${ }^{3}$ and Yonglun Luo ${ }^{3^{*}}$

\begin{abstract}
Introduction: Previously, we established a simple method for deriving mesenchymal stem cells (MSCs) from human induced pluripotent stem cells (iPSC-MSCs). These iPSC-MSCs were capable of forming osteogenic structures in scaffolds and nanofibers. The objective of this study is to systematically characterize the mesenchymal characteristics of the iPSC-MSCs by comparing them to bone marrow-derived MSCs (BM-MSCs).

Methods: Two iPSC-MSC lines (named as mRNA-iPSC-MSC-YL001 and lenti-iPSC-MSC-A001) and one BM-MSC line were used for the study. Cell proliferation, presence of mesenchymal surface markers, tri-lineage differentiation capability (osteogenesis, chondrogenesis, adipogenesis), and expression of "stemness" genes were analyzed in these MSC lines.

Results: The iPSC-MSCs were similar to BM-MSCs in terms of cell morphology (fibroblast-like) and surface antigen profile: CD29+, CD44+, CD73+, CD90+, CD105+, CD11b-, CD14-, CD31-, CD34-, CD45- and HLA-DR-. A faster proliferative capability was seen in both iPSC-MSCs lines compared to the BM-MSCs. The iPSC-MSCs showed adequate capacity of osteogenesis and chondrogenesis compared to the BM-MSCs, while less adipogenic potential was found in the iPSC-MSCs. The iPSC-MSCs and the tri-lineage differentiated cells (osteoblasts, chondrocytes, adipocytes) all lack expression of "stemness" genes: OCT4, SOX2, GDF3, CRIPTO, UTF1, DPPA4, DNMT3B, LIN28a, and SAL4.
\end{abstract}

Conclusions: The MSCs derived from human IPSCS with our method have advanced proliferation capability and adequate osteogenic and chondrogenic properties compared to BM-MSCs. However, the iPSC-MSCs were less efficient in their adipogenicity, suggesting that further modifications should be applied to our method to derive iPSC-MSCS more closely resembling the naive BM-MSCs if necessary.

\section{Introduction}

Mesenchymal stem cells (MSCs) have been widely used for tissue engineering due to their unique tri-lineage differentiation properties: osteogenesis, chondrogenesis, and adipogenesis $[1,2]$. One attractive application of MSCs for tissue engineering is to functionalize biomaterials scaffolds

\footnotetext{
* Correspondence: GQZHOU@SZU.EDU.CN; ALUN@BIOMED.AU.DK ${ }^{\dagger}$ Equal contributors

${ }^{4}$ Shenzhen Key Laboratory for Anti-aging and Regenerative Medicine, Health Science Center, Shenzhen University, 518060 Shenzhen, China ${ }^{3}$ Department of Biomedicine, the Health Faculty, Aarhus University, 8000 Aarhus C, Denmark

Full list of author information is available at the end of the article
}

by MSC co-culturing and differentiation. This can facilitate the replacement and integration of scaffolds into repairing tissues [3-5]. Currently, adult bone marrowderived mesenchymal stem cells (BM-MSC) seem to be the most reliable cell source for tissue engineering [6]. However, limited proliferating capability of the BMMSCs and their replicative senescence after in vitro expansion hamper their applications in a wide range of clinical applications [7].

Stem cell-derived MSCs have emerged as a significant alternative cell source to BM-MSCs. Human embryonic stem cells were mostly used for MSC derivation (ESC-MSCs), 
but concerns from ethical, pathological, and immunological perspectives have greatly limited the broad use of ESC-MSCs for clinical interventions. Induced pluripotent stem cells (iPSCs) generated from somatic cells represent a potentially inexhaustible cell resource with a pluripotent potential similar to ESCs. These iPSCs have the potential for patient-specific autologous therapies for diseases resulting from injury, genetic predisposition and/or aging. This approach eliminates most problems of immune incompatibility and ethical concerns. Since the first report of iPSC generation from mice and human fibroblasts by retrovirus-mediated reprogramming in 2006 and 2007 [8, 9], several methods have been established with improved safety and efficacy, such as protein transduction, mRNA reprogramming, and transgene free methods with a cocktail of small molecules [10-12].

Several attempts have been made to derive functional MSCs from iPSCs (iPSC-MSCs) [13-15]. These iPSCMSCs obtain most mesenchymal characteristics of the naïve BM-MSCs. They are positive for typical mesenchymal markers such as CD29 (integrin beta-1), CD73 (ecto-5'-nucleotidase), CD90 (Thy-1), CD105 (endoglin), and CD146 (cell surface glycoprotein MUC18). They lack expression of CD31 (platelet endothelial cell adhesion molecule-1), CD34 (hematopoietic progenitor antigen) and CD45 (leukocyte common antigen). Most importantly, these iPSC-MSCs are capable of differentiating into three functional lineages (osteoblasts, chondrocytes and adipocytes), and diminish histoincompatibilitymediated immune responses. Moreover, the iPSCMSCs show much greater proliferation and self-renewal capacity than BM-MSCs, which makes the iPSC-MSCs a promising substitute for BM-MSCs and ESC-MSCs. However, safety and efficacy still remain the major challenges for iPSC-MSCs.

To simplify the MSC generation procedure, we have previously established a method for iPSC-MSC derivation, which involves culturing the iPSCs directly in MSC differentiation medium followed by serial trypsinization-based passaging [16]. We have shown that the iPSC-MSCs derived by this method obtained a typical mesenchymal surface marker profile (CD73+, CD90+, CD105+, CD34-, $\mathrm{CD} 45-)$ and were capable of osteogenesis in tissue culture vessels, synthetic three-dimensional and nanofibers. They also lack tumorigenicity when transplanted into immunodeficient mice $[4,16]$.

Although the iPSC-MSCs derived by our method retain the mesenchymal functions, their multipotent efficacy as compared to naïve BM-MSCs is still unknown. To further characterize the efficacy of iPSC-MSCs derived by our method, we now compare the proliferative rate, mesenchymal marker profile (CD73+, CD90+, CD105+, CD34-, CD45-), tri-lineage differentiation capacity (osteogenesis, chondrogenesis, and adipogenesis), and pluripotency of two iPSC-MSC lines and one BM-MSC line.

\section{Methods}

\section{Cell culture}

Normal human dermal fibroblasts (NHDFs) were kindly provided by Prof. Thomas G. Jensen from the Department of Biomedicine, Aarhus University, Aarhus, Denmark. The use of anonymous NHDFs for basic research is conducted under the guidelines from the Danish Regional Committee on Health Research Ethics. All studies were conducted in compliance with the Helsinki Declaration (http://www.wma.net/en/30publications/10 policies/b3/index.html). NHDF were cultured in D10 medium consisting of Dulbecco's modified Eagle's medium (DMEM), $10 \%$ fetal calf serum, 2 mM L-glutamine, and $1 \%$ penicillin/streptomycin. All ethical approval and consent was received for the acquisition of these cells. Human iPSCs were cultured in knockout serum replacement (KSR) media, consisting of knockout DMEM (10829-018, Gibco), 20 \% Knock Out Serum Replacement (10828-028, Gibco), 2 mM L-glutamine, $1 \%$ penicillin/streptomycin, $0.1 \mathrm{mM}$ nonessential amino acids (11140-050, Gibco), $0.1 \mathrm{mM}$ 2-mercaptoethanol (313-50-010, Gibco) and $10 \mathrm{ng} / \mathrm{ml}$ human recombinant basic fibroblast growth factor (PHG0026, Invitrogen). The mRNA-iPSCs and mRNA-iPSC-MSC-YL001 were generated by mRNA transfection and characterized previously [16]. The human lenti-iPSCs were generated with lentivirus transduction using a single polycistronic lentiviral vector expressing OCT4, SOX2, KLF4 and c-MYC according to the methods reported by Papapetrou et al. [17]. Human BM-MSCs were purchased from Lonza (PT-2501) and cultured in MSC medium consisting of DMEM-low glucose (31885-023, Gibco), $10 \%$ fetal bovine serum (FBS; 26140-079, Gibco), 2 mM L-Glutamine and $1 \%$ penicillin/streptomycin. The K562 cells were kindly provided by Marianne Hokland from the Department of Biomedicine, Aarhus University. All cells were cultured in a tissue culture incubator with $5 \% \mathrm{CO}_{2}$ at $37^{\circ} \mathrm{C}$.

\section{Lentivirus packaging}

HEK293 cells were cultured in D10 medium. At the day of transfection, $1 \times 10^{7}$ HEK293 cells in each P15 dish (nine dishes in total) were transfected by the $\mathrm{CaPO}_{4}$ coprecipitation method with pRSV-REV, pMD.2G, pMDGP$\mathrm{Lg} / \mathrm{pRRE}$ plasmids and a lentiviral vector (pLM-fSV2A, Addgene ID 27512 [18]) expressing the four Yamanaka factors (OCT4, KLF4, c-Myc and SOX2) polycistronically. One day after transfection, cells were fed with fresh medium (17 ml/dish). Cell medium containing lentivirus was harvested at $48 \mathrm{~h}$ and $72 \mathrm{~h}$ post-transfection. Lentivirus was concentrated by ultra-centrifugation $\left(25,000 \mathrm{rpm}, 4{ }^{\circ} \mathrm{C}\right.$, 
L7 Ultracentrifuge, Beckman). Virus pellets were dissolved with phosphate-buffered saline (PBS) and stored at $-80{ }^{\circ} \mathrm{C}$. Virus titer was measured with the P24 Elisa kit (XB-1000, XpressBio).

\section{Lentivirus-mediated reprogramming}

NHDFs $\left(1.5 \times 10^{5}\right.$ cells/per well $)$ were seeded in a sixwell plate 1 day before transduction. Cells were transduced with reprogramming lentivirus in the presence of polybrene $(8 \mu \mathrm{g} / \mathrm{mL})$ in D10 medium. Cell media were changed every other day. Six days post-transduction, transduced NHDFs $\left(2 \times 10^{4}\right.$ cells/per well $)$ were harvested by trypsinization and seeded on irradiationinactivated mouse feeder cells in six-well plates, and cultured in KSR medium. KSR medium was changed daily. Approximately 21 days post-transduction, the iPSC colonies were ready for picking and expansion.

\section{Immunofluorescence staining}

For immunofluorescence staining, cells were fixed in $4 \%$ paraformaldehyde for $20 \mathrm{~min}$, followed by PBS wash (three times, $5 \mathrm{~min}$ each) and permeabilization with $0.3 \%$ Triton X-100 in PBS for 10 min. The cells were then blocked with blocking solution (5\% donkey serum in PBS) at room temperature for $30 \mathrm{~min}$ and incubated with the primary antibodies overnight at $4{ }^{\circ} \mathrm{C}$. Goat antihuman OCT3/4 (Abcam, ab27985, 100× diluted) and rabbit antihuman Nanog (Abcam, ab80892, 100× diluted) were used. Cells were then stained with a secondary antibody for $2 \mathrm{~h}$. Alexa 594 donkey anti-goat IgG $(\mathrm{H}+\mathrm{L})$ and Alexa Fluor 488 Donkey Anti-Rabbit IgG ( $\mathrm{H}+\mathrm{L})$ (Life Technologies) were used for second antibody staining. For live cell staining of TRA-1-60 and CD44, cells were stained using the live cell imaging kit from Life Technologies (Tra-1-60 AF594, CD44 AF488) according to the manufacturer's protocol. All images were taken with a Leica fluorescence microscope.

\section{Derivation of MSCs from iPSCs generated by lentiviral reprogramming}

The iPSC-MSC derivation was performed according to our previous protocol. One characterized pluripotent lenti-iPSC line was used for MSC differentiation. Briefly, 3 days after passaging the lenti-iPSCs to feeder cell culture, the KSR medium was replaced with MSC medium. The lenti-iPSCs were maintained in MSC medium for 2 weeks, with medium changed every other day. Subsequently, cells were passaged to gelatin-coated $(0.1 \%$ gelatin, room temperature for $2 \mathrm{~h}$ ) tissue culture vessels by trypsinization ( $0.25 \%$ trypsin/1 mM EDTA). Cells were defined as passage 1 (P1) after the first passaging. For maintenance of iPSC-MSCs, cells were passaged when $90 \%$ confluent and seeded with a density of $1.6 \times 10^{4}$ cells $/ \mathrm{cm}^{2}$ to new tissue culture vessels.
MSC surface marker characterization by flow cytometry

Detail information on antibodies against the human antigens CD11b, CD14, CD29, CD31, CD34, CD44, CD45, CD73, CD90, CD105 and HLA-DR are shown in Table 1. Cells were harvested by trypsinization and washed with $2 \%$ FBS-PBS twice; $2 \times 10^{5}$ cells were re-suspended in $100 \mu \mathrm{l} 2 \%$ FBS-PBS and incubated with the conjugated antibody for $30 \mathrm{~min}$ at room temperature in the dark. Stained cells were then washed with $2 \%$ FBS-PBS twice and re-suspended in $500 \mu \mathrm{l} 1 \%$ formaldehyde-PBS for flow cytometry analysis (LSRFortessa); 10,000 events were recorded for each sample and data were analyzed with Flowjo.

\section{Number of population doublings}

BM-MSCs, mRNA-iPSC-MSC-YL001 and lenti-iPSCMSC-A001 were serially subcultured in six-well plates for 2 weeks. Briefly, cells were cultured in six-well plates; when cells were $90 \%$ confluent, they were trypsinized and counted with a hemocytometer. The cells were then cultured in a new six-well plate with the same starting cell density. The number of population doublings (NPD) was calculated according to the following equation: NPD = $\log 10(\mathrm{Nh} / \mathrm{Ni}) \times 3.33$, where $\mathrm{Nh}$ and $\mathrm{Ni}$ are the numbers of harvested and initiating cells, respectively [19]. NPDs were performed in six replicates for each type of MSC.

\section{Cell proliferation rate assay}

Cell proliferation rate measurements were conducted using the Click-iT ${ }^{\ominus}$ EdU Alexa Fluor ${ }^{\circ} 488$ Flow Cytometry Assay Kit (Cat. no. C10425, Life Technologies). K562, BM-MSCs, mRNA-iPSC-MSC-YL001, and lentiiPSC-MSC-A001 cells were seeded in six-well plates $(1.0 \times 105$ cells/well $) 24 \mathrm{~h}$ prior to changing to fresh growth medium (negative control) or growth medium containing $10 \mu \mathrm{M}$ EdU. Cells cultured in growth medium without EdU were used as negative controls. Cells were incubated at $37^{\circ} \mathrm{C}$ in a humidified incubator

Table 1 List of flow cytometry antibodies

\begin{tabular}{lll}
\hline Antibody & Catalogue number & Dilution \\
\hline CD11b FITC & eBioscience, 11-0113 & $40 \times$ \\
CD14 V450 & BD, 560350 & $40 \times$ \\
CD29 APC & eBioscience, 17-0299 & $40 \times$ \\
CD31 V450 & BD, 561653 & $40 \times$ \\
CD34 APC & BD, 560940 & $10 \times$ \\
CD44 Cy5.5 & BD, 560531 & $40 \times$ \\
CD45 V450 & BD, 560368 & $40 \times$ \\
CD73 PE & BD, 561014 & $10 \times$ \\
CD90 FITC & BD, 555595 & $50 \times$ \\
CD105 Cy5.5 & BD, 560819 & $40 \times$ \\
HLA-DR Cy5.5 & BD, 560652 & $40 \times$ \\
\hline
\end{tabular}


with $5 \% \mathrm{CO}_{2}$ for $2 \mathrm{~h}$, then harvested by trypsinization and subjected to EdU staining according to the manufacturer's protocol. Briefly, cells were washed with $500 \mu \mathrm{L}$ $1 \%$ bovine serum albumin (BSA)-PBS, incubated with $100 \mu \mathrm{L}$ Click-iT fixative for $15 \mathrm{~min}$ in the dark, washed with $500 \mu \mathrm{L} 1 \%$ BSA-PBS, re-suspended with $100 \mu \mathrm{L}$ $1 \times$ component $\mathrm{E}$, stained in labeling cocktail for $30 \mathrm{~min}$, washed with $500 \mu \mathrm{L} 1 \times$ component $\mathrm{E}$, and analyzed by flow cytometry (LSRFortessa); 10,000 events were recorded for each sample and data were analyzed with Flowjo.

\section{Tri-lineage differentiation}

BM-MSCs, mRNA-iPSC-MSC-YL001, and lenti-iPS-MSCA001 cells (all P9) were used for the tri-lineage differentiation (osteogenesis, chondrogenesis and adipogenesis).

For osteogenic differentiation, cells were seeded at a density of $1.2 \times 10^{4}$ cells $/ \mathrm{cm}^{2}$ in 96 -well tissue culture plates. After 3 days, the media were changed to either MSC growth medium (as control) or osteogenic differentiation medium, which contains DMEM-high glucose (Invitrogen), 10 \% FBS (Gibco), 1 \% penicillin/streptomycin, $100 \mathrm{nM}$ dexamethasone (D2915, Sigma), $10 \mathrm{mM}$ $\beta$-Glycerophosphate (G9891, Sigma), $50 \mu \mathrm{M}$ ascorbic acid-2 phosphate (A8960, Sigma), and $10 \mu \mathrm{M}$ Vit-D (D1530, Sigma). Cells were maintained in growth medium or osteogenic differentiation medium for 3 weeks with medium changed every 3 days. At the end of differentiation, samples were subjected to Alizarin Red S (Sigma) staining, calcium quantification analysis following the manufacturer's instructions, and quantitative PCR analysis.

For chondrogenic differentiation, $2.5 \times 10^{5}$ cells were centrifuged at $500 \mathrm{~g}$ for $10 \mathrm{~min}$ to form a small pellet in a "V" shape 96-well plate. The cell pellets were cultured in either MSC growth medium (as control) or chondrogenic differentiation medium consisting of DMEM (F12, Gibco) supplemented with $10 \% \mathrm{FBS}, 50 \mu \mathrm{g} / \mathrm{mL}$ ascorbic acid, $100 \mathrm{nM}$ dexamethasone, 1:100 ITS premix (BD Biosciences), $40 \mu \mathrm{g} / \mathrm{ml} \mathrm{L}$-proline (Sigma-Aldrich), $10 \mathrm{ng} / \mathrm{mL}$ human recombinant TGF- $\beta 3$ (R\&D systems), and $1 \%$ penicillin/streptomycin $[16,20]$. The medium was changed every 3 days. After 3 weeks, cell pellets were collected for toluidine blue staining to validate the extracellular chondrocyte matrix [21]. Purple stain of proteoglycans was observed by phase-contrast microscopy.

Adipogenic differentiation was carried out by culturing the cells at a density of $1.2 \times 10^{4}$ cells $/ \mathrm{cm}^{2}$ in DMEMhigh glucose (Invitrogen) supplemented with $15 \%$ horse serum (Sigma), and $100 \mathrm{nM}$ dexamethasone (Sigma), $1 \%$ penicillin/streptomycin (MSC growth medium as control). After 3 weeks, cells were stained with Oil-red O (Sigma-Aldrich) solution to evaluate differentiation using standard techniques as previously described [22].

\section{PCR and quantitative PCR}

Total RNA was extracted with an RNeasy Plus Mini Kit (Qiagen) according to the manufacturer's instructions, followed by quantification with a Nanodrop spectrophotometer and qualification by $1 \%$ agarose gel electrophoresis. First-strand cDNA was synthesized with approximately $50 \mathrm{ng}$ of total RNA using a cDNA synthesis kit (170-8891, Bio-Rad). PCR primers for stemness genes and osteogenic genes are listed in Table 2.

For PCR characterization of the expression of stemness genes (OCT4, KLF4, C-MYC, SOX2, GDF3, CRIPTO, UTF1, DPPA4, DNMT3B, LIN28A and SALL4), PCR was performed with pfx DNA Polymerase (11708-039, Invitrogen) using $2 \mu \mathrm{l}$ (five times diluted) cDNA as DNA temperate. The following PCR program was optimized for each stemness gene: 1 cycle of $94{ }^{\circ} \mathrm{C}$ for $3 \mathrm{~min} ; 30$ cycles of $94{ }^{\circ} \mathrm{C}$ for 30 seconds, $60{ }^{\circ} \mathrm{C}$ or $58{ }^{\circ} \mathrm{C}$ or $53{ }^{\circ} \mathrm{C}$ (see Table 2) for 30 seconds, and $68^{\circ} \mathrm{C}$ for 30 seconds; 1 cycle at $68{ }^{\circ} \mathrm{C}$ for $7 \mathrm{~min}$. The GAPDH gene was used as an endogenous control.

For quantitative PCR of osteogenic genes (of runtrelated transcription factor 2 (RUNX2), alkaline phosphatase $(A L P)$, collagen type I alpha 1 (COL1A1), and osteocalcin $(O C)$ ), $2 \mathrm{uL}$ (five times diluted) cDNA was used. Quantitative PCR was performed using the LightCycle 480 SYBR Green I Master kit on LightCycler 480 (Roche) with the following PCR program: 1 hold at $95^{\circ}$ C for $5 \mathrm{~min} ; 50$ cycles at $95{ }^{\circ} \mathrm{C}$ for 10 seconds, $60{ }^{\circ} \mathrm{C}$ for 10 seconds, and $72{ }^{\circ} \mathrm{C}$ for 15 seconds. To mask the signal from primer dimers, fluorescent signal was measured at $77{ }^{\circ} \mathrm{C}$ for $R U N X 2,80{ }^{\circ} \mathrm{C}$ for $A L P, 82{ }^{\circ} \mathrm{C}$ for GAPDH and COL1A1, and $84^{\circ} \mathrm{C}$ for $O C$. Relative gene expression was calculated using the $2^{-\Delta \Delta c t}$ method after normalization to the reference gene GAPDH.

\section{Statistics}

All values are reported as mean \pm SD. Data were checked for normal distribution using QQ-plots. The osteogenic gene expression between growth and osteogenic media were compared by $t$-test in each cell line. Quantitative calcium deposition was investigated using two-way analysis of variance (media type $\times$ cell line). If interactions were observed, the variables were investigated separately by the Scheffe method. $P<0.05$ was considered statistically significant.

\section{Results}

MSCs from iPSCs induced by lentiviral-mediated reprogramming have the same fibroblast-like morphology as BM-MSCs

The procedure for iPSC-MSC derivation, which we had established previously, was based on an iPSC line established by mRNA reprogramming from human fibroblasts 
Table 2 List of primers

\begin{tabular}{|c|c|c|c|}
\hline Gene & Primer sequences $\left(5^{\prime}-3^{\prime}\right)$ & Annealing temperature $\left({ }^{\circ} \mathrm{C}\right)$ & Amplicon (bp) \\
\hline OCT4-F & CCTCACTTCACTGCACTGTA & 58 & 163 \\
\hline OCT4-R & CAGGTITTCTITCCCTAGCT & & \\
\hline KLF4-F & GATGAACTGACCAGGCACTA & 60 & 144 \\
\hline KLF4-R & GTGGGTCATATCCACTGTCT & & \\
\hline C-MYC-F & TGCCTCAAATTGGACTTTGG & 58 & 191 \\
\hline C-MYC-R & GATTGAAATTCTGTGTAACTGC & & \\
\hline SOX2-F & CCCAGCAGACTTCACATGT & 58 & 150 \\
\hline$S O \times 2-R$ & CCTCCCATTTCCCTCGTTT & & \\
\hline GDF3-F & AAATGTTTGTGTTGCGGTCA & 53 & 178 \\
\hline GDF3-R & TCTGGCACAGGTGTCTTCAG & & \\
\hline CRIPTO-F & CGGAACTGTGAGCACGATGT & 58 & 65 \\
\hline CRIPTO-R & GGGCAGCCAGGTGTCATG & & \\
\hline UTF1-F & CCGTCGCTGAACACCGCCCTGCTG & 58 & 179 \\
\hline UTF1-R & CGCGCTGCCCAGAATGAAGCCCAC & & \\
\hline DPPA4-F & GGAGCCGCCTGCCCTGGAAAATTC & 53 & 407 \\
\hline DPPA4-R & TITTCCTGATATTCTATTCCCAT & & \\
\hline DNMT3B-F & TGCTGCTCACAGGGCCCGATACTTC & 58 & 170 \\
\hline DNMT3B-R & TCCTTTCGAGCTCAGTGCACCACAAAAC & & \\
\hline LIN28-F & AGTAAGCTGCACATGGAAGG & 58 & 420 \\
\hline LIN28-R & ATTGTGGCTCAATTCTGTGC & & \\
\hline SALL4-F & GCCGTGAAGACCAATGAGAT & 58 & 243 \\
\hline SALL4-R & СTCCTTCCACGCAAGTTCTC & & \\
\hline RUNX2-F & CAGTAGATGGACCTCGGGAA & 60 & 188 \\
\hline RUNX2-R & CCTAAATCACTGAGGCGGTC & & \\
\hline ALP-F & ATCAGGGACATTGACGTGATC & 60 & 137 \\
\hline ALP-R & TTCCAGGTGTCAACGAGGTC & & \\
\hline COL1A1-F & AGGGCCAAGACGAAGACATC & 60 & 138 \\
\hline COL1A1-R & AGATCACGTCATCGCACAAC & & \\
\hline OC-F & AGTCCAGCAAAGGTGCAGCC & 60 & 169 \\
\hline OC-R & TCAGCCAACTCGTCACAGTC & & \\
\hline GAPDH-F & TGGTATCGTGGAAGGACTCATGAC & 53 & 189 \\
\hline GAPDH -R & ATGCCAGTGAGCTTCCCGTTCAGC & & \\
\hline
\end{tabular}

[16]. It is unknown whether this procedure could be applied to derive functional MSCs from iPSC lines generated by other methods. Lentiviral-mediated reprogramming is favored for its high reprogramming efficiency relative to other methods [17]. We firstly generated one iPSC line using lentiviral-mediated reprogramming. The iPSC line exhibited typical stem cell morphology; expressed pluripotent cell cells markers: TRA1-60 (podocalyxin), OCT4 (octamer-binding transcription factor 4, also known as POU domain, class 5, transcription factor 1), and NANOG (Nanog homeobox) (Fig. 1a); lacked expression of CD44 (the absence of which is crucial for iPSC pluripotency (Fig. 1a)) [23]; and retained normal polyploidy (diploid as fibroblasts, Fig. 1b). We differentiated the iPSC colony into MSCs using our preciously established procedure [16]. The MSCs derived from lentivial reprogramming iPSCs (lenti-iPSC-MSCs-A001) had the same morphology (fibroblast-like) as the BM-MSCs and the mRNA-iPSC-MSC-YL001 line reported previously [16] (Fig. 1c), and are karyotypically normal (Fig. 1d).

The iPSC-MSCs obtained similar mesenchymal surface marker profiles to BM-MSCs through in vitro cultivation To verify whether the iPSC-MSCs harbor mesenchymal characteristics, we analyzed the expression of five MSCpositive surface markers-CD29 (Integrin beta-1), CD44 


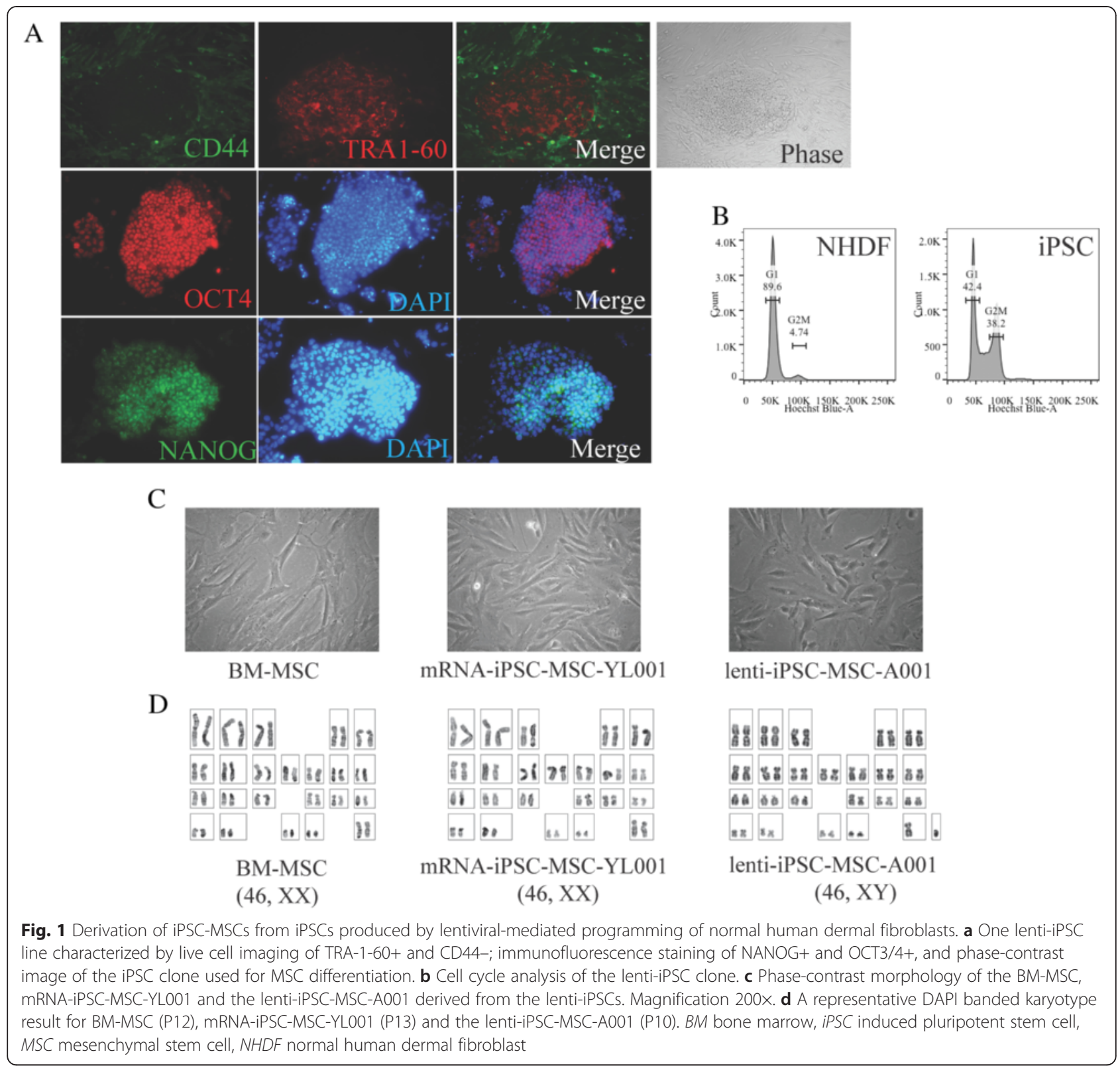

(Phagocytic glycoprotein 1), CD73 (ecto-5'-nucleotidase), CD90 (Thy-1), and CD105 (endoglin) - and six MSCnegative surface markers-CD11b (integrin alpha-M), CD14 (monocyte differentiation antigen), CD31 (platelet endothelial cell adhesion molecule), CD34 (hematopoietic progenitor cell antigen), CD45 (T-cell surface glycoprotein), and HAL-DR (major histocompatibility complex class II antigen)-in lenti-iPSC-MSC-A001 (P10), mRNAiPSC-MSC-YL001 (P14) and the BM-MSCs (P13) by flow cytometry. As shown in Fig. 2, the mRNA-iPSC-MSCYL001, lenti-iPSC-MSC-A001 and BM-MSCs lacked expression of CD11b, CD14, CD31, CD34, CD45 and HLA-DR. Almost $100 \%$ of the lenti-iPSC-MSC-A001 and mRNA-iPSC-MSC-YL001 cells were positive for CD29,
CD44, and CD73, similar to that for BM-MSCs. Robust expression of CD90 and CD105 (almost $100 \%$ positivity) was found in BM-MSCs. The abundance of CD90- and CD105-positive cells in the lenti-iPSC-MSC-A001 cells was increased following in vitro cultivation. At P3, about $70 \%$ and $80 \%$ of the cells were positive for CD90 and CD105, respectively (data not shown). At P13, the lenti-iPSC-MSC-A001 cells were approximately $98.15 \%$ CD90 positive and $85.64 \%$ CD105 positive (Fig. 2). Among the mRNA-iPSC-MSC-YL001 cells, robust CD90 and CD105 expression (89.18\% and $87.47 \%$ positivity at P14, respectively) were observed, which is consistent with our previous observation [16]. Thus, it appears that lenti-iPSC-MSC-A001 (late passage) and 


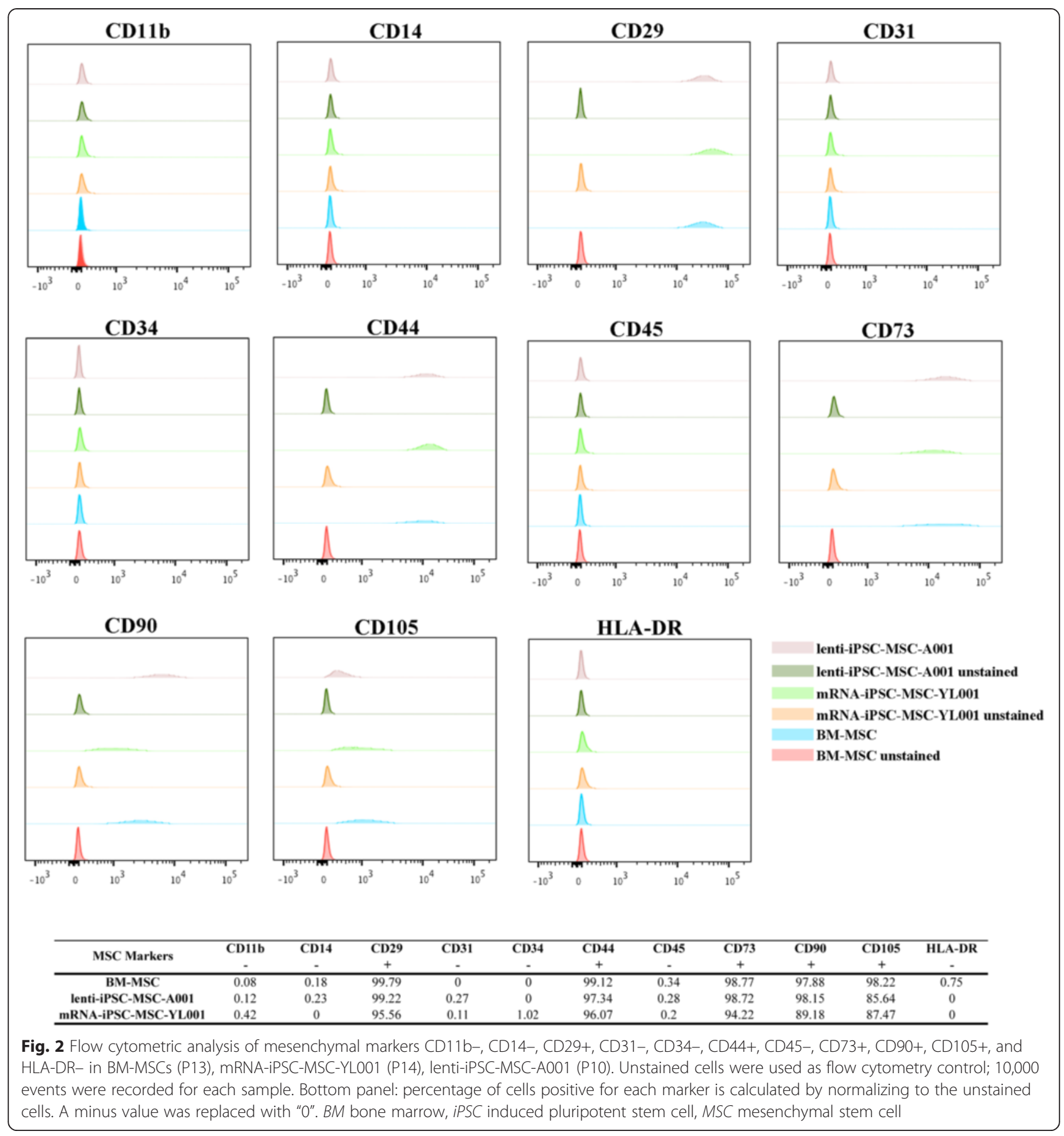

mRNA-iPSC-MSC-YL001 cells display similar mesenchymal surface marker profiles as BM-MSCs. However, the MSC surface marker characterization also indicates the heterogeneity of iPSC-MSCs derived by our method, with approximately $10 \%$ cells negative for CD105 (Fig. 2). Based on the finding of passagedependent mesenchymal enrichment in the lenti-iPSCMSC-A001, cells with over 6 passages were used for the following functional characterizations.
The iPSC-MSCs possess fast proliferative capability

The iPSC-MSCs have become a promising substitute for BM-MSCs or MSCs derived from other adult tissues. The advantage of using iPSC-MSCs is attributed to their higher self-renewal capability. We compared the proliferation rate of the two iPSC-MSC lines to BM-MSCs using the Click IT-based proliferation assay and flow cytometry. K562 cancer cells were used a positive control for the assay (55.7 \% proliferation rate, Fig. 3a). Higher 


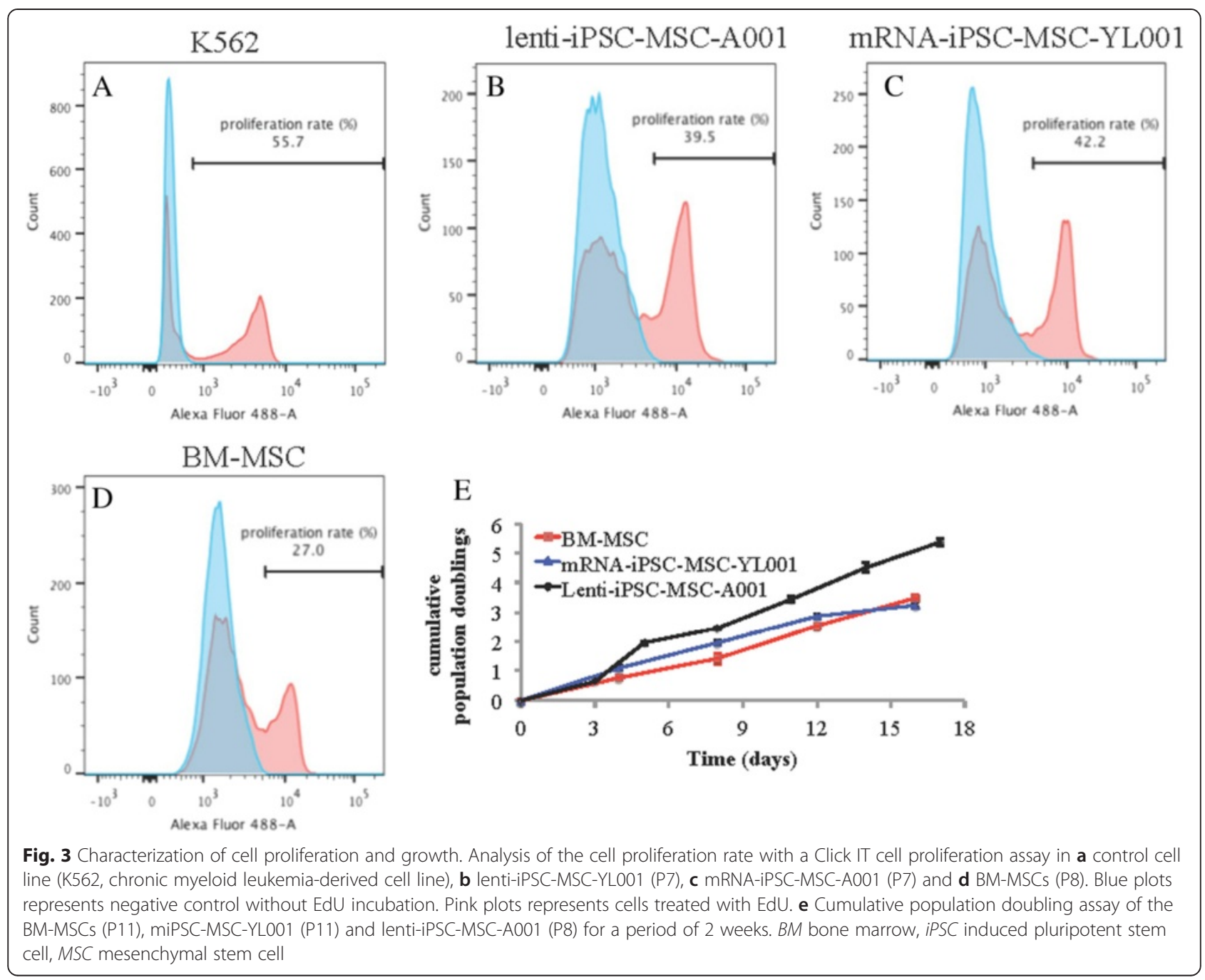

proliferation rate was observed in the mRNA-iPSCMSC-YL001 (42.2 \%, Fig. 3b) and lenti-iPSC-MSC-A001 (39.5 \%, Fig. 3c) than the BM-MSCs (27 \%, Fig. 3d). The fast proliferation capacity of iPSC-MSCs was further validated by population doubling array (Fig. 3e).

iPSC-MSCs possess adequate osteogenic and chondrogenic, but less adipogenic capabilities, compared with BM-MSCs

According to the International Society for Cellular Therapy, the capability of tri-lineage differentiation in vitro is one of the major criteria for defining MSCs [24]. We have previously shown that the mRNA-iPSC-MSCYL001 line is capable of tri-lineage differentiation, with a focus on osteogenesis on synthetic biocompatible scaffolds [16]. However, the efficacy of tri-lineage differentiation as compared to BM-MSCs is yet to be addressed. Thus, we compared the osteogenic, chondrogenic and adipogenic differentiation capabilities of the two iPSCMSC lines relative to the BM-MSC line.

After 3 weeks of osteogenic induction, calcium mineralization was observed in all three MSC lines by Alizarin Red S staining (Fig. 4a). Mineralization was not observed in the MSCs cultured in normal growth medium. A more homogeneous and robust calcium staining was seen in the lenti-iPSC-MSC-A001 than in the mRNA-iPSC-MSC-YL001. This observation was further confirmed by quantification of calcium content (Fig. 4b). The lenti-iPSC-MSC-A001 line has an efficacy of osteogenesis similar to BM-MSCs. However, approximately twofold less osteogenic capacity was found in the mRNAiPSC-MSC-YL001 cultures, which is likely due to less positivity of CD90 (Fig. 2) [25]. Increased expression of four osteogenic genes (runt-related transcription factor 2 (RUNX2), alkaline phosphatase $(A L P)$, collagen type I alpha 1 (COL1A1), and osteocalcin $(O C)$ ) was detected in all MSCs after 3 weeks of osteogenic differentiation 


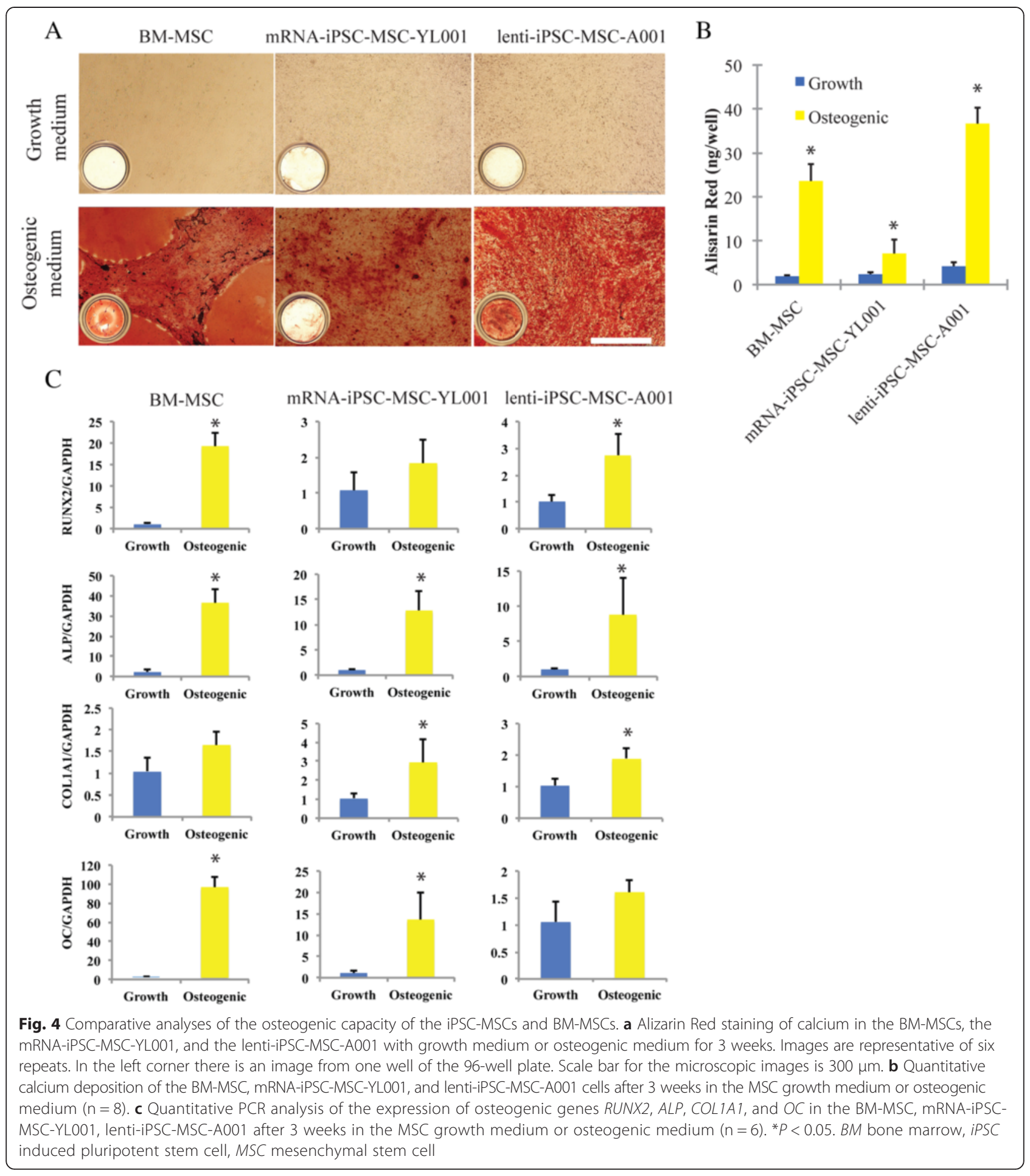

(Fig. 4c). The induced expression of RUNX2, $A L P$ and $O C$ was more robust in BM-MSCs relative to the iPSCMSCs, whereas Colla1 was more strongly expressed in the iPSC-MSC cultures. Taken together, these results suggest that iPSC-MSCs derived with our method can achieve an adequate osteogenic efficacy similar to that of BM-MSCs.
For chondrogenic differentiation, the lenti-iPSC-MSCA001, mRNA-iPSC-MSC-YL001 and BM-MSC lines were grown in a "V" shape 96-well plate according to a protocol described previously [26]. Toluidine blue staining of pellet sections was used to assess chondrogenic differentiation. Cartilaginous extracellular matrix is stained purple while undifferentiated or fibrous tissue is stained blue. After 
3 weeks of chondrogenic differentiation, cartilaginous extracellular matrix was detected in all MSC lines cultured in chondrogenic medium (Fig. $5 \mathrm{~d}-\mathrm{f}$ ), but not in growth medium (Fig. 5a-c). The lenti-iPSC-MSC-A001 and mRNA-iPSC-MSC-YL001 had formed similar cartilaginous structures compared with the BM-MSCs.
The adipogenic potential of the iPSC-MSCs was also characterized by in vitro adipogenic differentiation. Lipid droplets formed by adipogenesis were assessed by Oil Red $\mathrm{O}$ staining. After 3 weeks of adipogenic differentiation, lipid droplets were formed in both iPSC-MSC lines and the BM-MSCs in the adipogenic differentiation
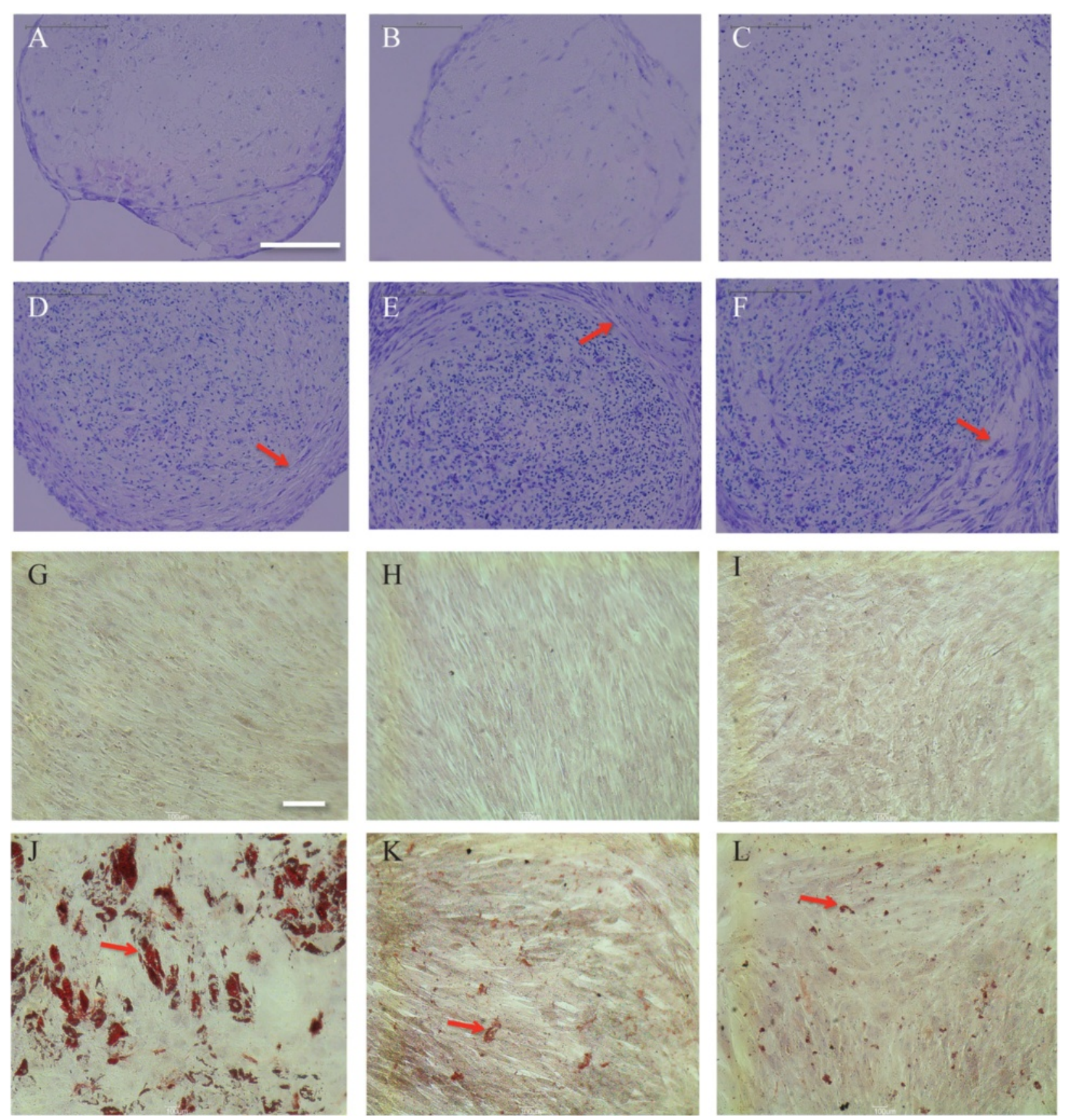

Fig. 5 Comparative analysis of the chondrogenic and adipogenic capacities. Toluidine blue staining of cartilaginous extracellular matrix of the BM-MSCs (a and $\mathbf{d}$ ), the mRNA-iPSC-MSC-YL001 (b and $\mathbf{e}$ ), and the lenti-iPSC-MSC-A001 (c and $\mathbf{f}$ ) after 3 weeks in MSC growth medium (a-c) or chondrogenic medium (d-f). Oil-Red O staining of lipids in the BM-MSCs ( $\mathbf{g}$ and $\mathbf{j}$ ), the mRNA-iPSC-MSC-YL001 (h and $\mathbf{k}$ ), and the lenti-iPSC-MSC-A001 (i and $\mathbf{I})$ after 3 weeks in MSC growth medium $(\mathbf{g}-\mathbf{i})$ or adipogenic medium $(\mathbf{j}-\mathbf{I})$. Arrow heads indicate the cartilaginous extracellular matrix (d-f) and lipids (j-l). Scale bar: $\mathbf{a}-\mathbf{f}, 150 \mu \mathrm{m} ; \mathbf{g}-\mathbf{I}, 300 \mu \mathrm{m}$ 
medium (Fig. 5j-l) but not in the growth medium (Fig. 5g-i). However, the adipogenic efficacy of both iPSC-MSC lines was much lower than the BM-MSCs. Lipid droplets from iPSC-MSCs were much smaller than those from BM-MSCs.

The iPSC-MSCs and tri-lineage differentiated cells lack expression of stemness genes

Loss of "stemness" in iPSC-MSCs and iPSC-MSCderived lineages is one crucial safety criterion when applying iPSC-MSCs to tissue engineering and cell therapy. Lack of tumorigenic potential of the mRNA-iPSC-MSCYL001 line was validated previously [16]. We further characterized the loss of "stemness" in the iPSC-MSC lines by analyzing the expression of 11 stemness genes (OCT4, KLF4, C-MYC, SOX2, GDF3, CRIPTO, UTF1, $D P P A 4, D N M T 3 B$, LIN28A and SALL4) in the original iPSCs, iPSC-MSCs, and tri-lineage differentiated cells (osteogenesis, chondrogenesis, and adipogenesis). All stemness genes were expressed in the iPSCs, whereas most stemness genes, except $K l f 4$ and $C$-Myc, were not expressed in the iPSC-MSCs and the tri-lineage differentiated cells (Fig. 6). We also found that Klf4 and $c-M y c$ were expressed in the NHDF, which is similar to what has been reported previously $[8,27]$.

\section{Discussion}

With technical improvements in generating pluripotent stem cells, differentiation of iPSCs to functionally specific cell types remains a major challenge. MSCs are a promising cell type for personalized therapies such as tissue engineering [28], immunosuppressive biotherapy [29], and regenerative organ repair [30]. We have previously developed a simple protocol to derive iPSC-MSCs and applied them for bone tissue engineering in synthetic three-dimensional scaffolds and nanofibers $[4,16]$.

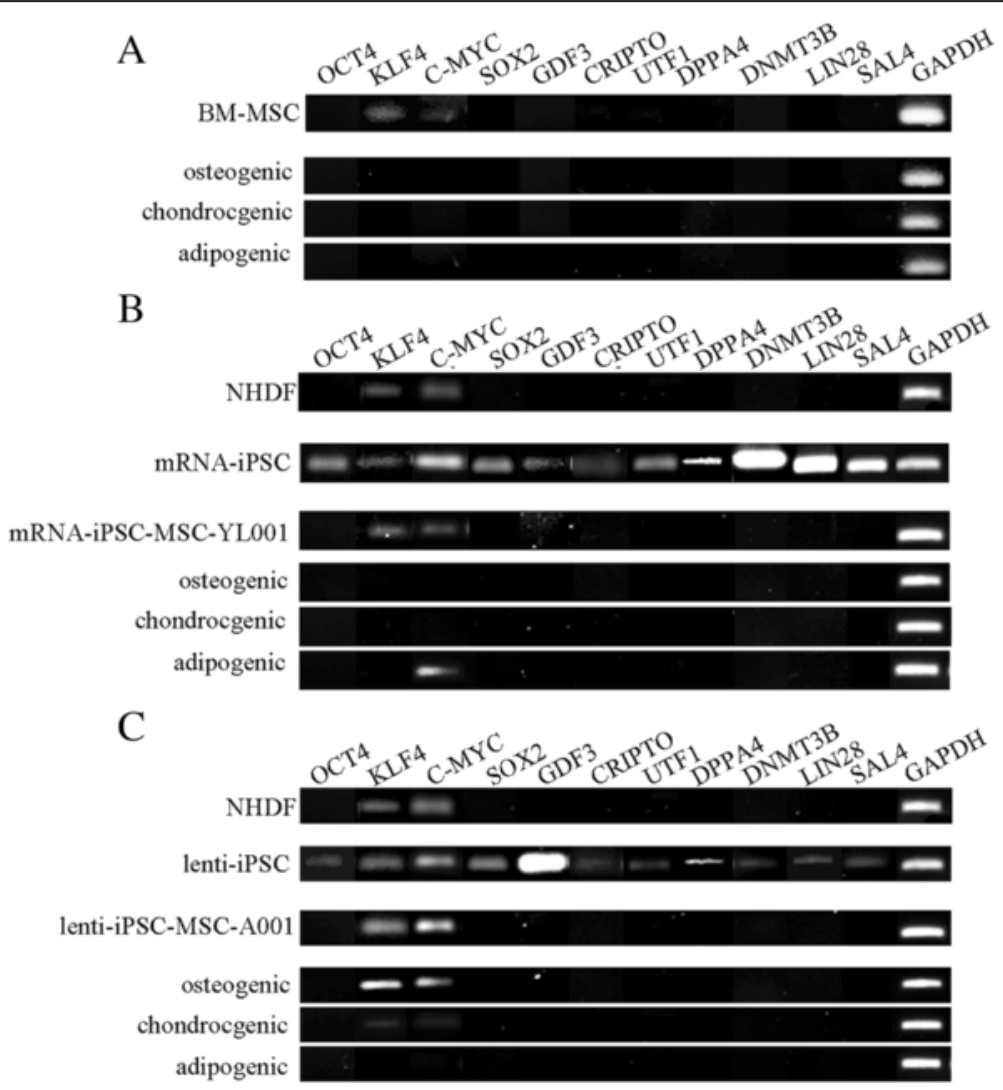

Fig. 6 Gene expression analysis of "stemness" genes in the NHDFs, iPSCs (two lines: mRNA-iPSC and lenti-iPSC), BM-MSCS, mRNA-iPSC-MSC-YL001, lenti-iPSC-MSC-A001 and the three derived lineages (osteogenic, chondrogenic, adipogenic). Expression of eleven stemness genes (POU class 5 homeobox 1 (OCT4/ POU5F1), Kruppel-like factor 4 (KLF4), v-myc avian myelocytomatosis viral oncogene homolog (C-MYC), SRY (sex determining region Y)-box 2 (SOX2), growth differentiation factor 3 (GDF3), teratocarcinoma-derived growth factor 1 (CRIPTO/ TDGF1), undifferentiated embryonic cell transcription factor 1 (UTF1), developmental pluripotency associated 4 (DPPA4), DNA (cytosine-5-)-methyltransferase 3 beta (DNMT3B), lin-28 homolog A (LIN28A) and spalt-like transcription factor 4 (SALL4)) was analyzed by PCR. a BM-MSCs in growth medium, and after 3 weeks in osteogenic, chondrogenic and adipogenic medium; $\mathbf{b}$ NHDF, mRNA-iPSCs, mRNA-iPSC-MSC-YL001 in growth medium, and mRNA-iPSC-MSC-YL001 after 3 weeks in osteogenic, chondrogenic and adipogenic medium; c NHDF, lenti-iPSCs, lenti-iPSC-MSC-A001 in growth medium, and IviPSC-MSC-A001 after 3 weeks in osteogenic, chondrogenic and adipogenic medium. BM bone marrow, iPSC induced pluripotent stem cell, MSC mesenchymal stem cell, NHDF normal human dermal fibroblast 
Here, we compared two iPSC-MSC lines (derived with our protocol) to naïve BM-MSCs. This study demonstrated that the iPSC-MSCs derived by our protocol display many similar characteristics relative to the BMMSCs, including fibroblast-like morphology, expression of typical mesenchymal markers, the capacity to efficiently differentiate into osteogenic and chondrogenic lineages, and loss of pluripotency. This study further supports our previous finding of efficient osteogenic potential for iPSC-MSCs in three-dimensional scaffolds and nanofibers $[4,16]$.

There are several advantages of using iPSC-MSCs for MSC-based therapies as compared to primary MSCs. The high proliferative capability iPSC-MSCs proven by this study (Fig. 3) and others [31-33], as well as the infinite self-renewal capacity of iPSCs [8], increase the potential of MSC-based cell therapies, which commonly requires repetitive administration of a large number of MSCs. Although MSCs can be easily isolated from various sources of adult tissues, such as BM, fat, placenta, blood, spleen, heart, pancreas, joints and so forth [34-36], this repetitive MSC isolation procedure would cause pain and risk of infection for patients. Furthermore, for certain patients with inherited genetic problems or age-related disorders, iPSC-MSCs would represent the best solution for gene- and cell-based therapy. One such example is Osteogenesis imperfect (OI), which is caused by dominant mutations of the type one collagen genes. With an adeno-associated virus-mediated gene targeting strategy, Deyle et al. generated iPSC-MSCs derived from OI patients and subsequently inactivated the mutated COL1A1 and COL1A2 genes in the iPSC-MSCs. The targeted iPSC-MSCs were able to restore normal collagen production and bone formation [37].

In terms of osteogenic and chondrogenic capacities of the iPSC-MSCs, we now have further confirmed their adequate efficacy relative to BM-MSCs $[4,16]$. Although only the osteogenicity was thoroughly investigated in this study due to our research focus, the chondrogenicity of iPSC-MSCs has been systematically confirmed by studies from other research groups [38, 39].

However, this study also showed that both iPSC-MSC lines were less efficient with respect to adipogenic differentiation relative to their osteogenicity and chondrogenicity (Fig. 5). Although both iPSC-MSC lines derived by our protocol were capable of adipogenesis [16], the adipocytes and lipid droplets were much smaller in the cultures of iPSC-MSCs compared with those of BMMSCs. Similarly, iPSC-MSCs derived by other methods have been reported to have less adipogenicity than BMMSCs $[32,40]$. A similar observation was reported for MSCs derived from human ESCs [41]. It is still unknown why MSCs derived from iPSCs or ESCs show less commitment towards adipogenicity. Many intrinsic factors could affect the adipogenicity of iPSC-MSCs, such as the iPSC-MSC derivation and cultivation methods, iPSC-MSC purity, and tissue of origin. Hynes et al. recently compared iPSC-MSCs originating from three different tissues: gingiva, periodontal ligament, and lung [42]. Although the efficacy of adipogenesis of those iPSC-MSCs was not assessed in their study, various capacities were observed among the three iPSC-MSCs lines, suggesting the notion that the tissue of origin plays a vital role in commitment differentiation. In our study, the lenti-iPSC-MSC-A001 had a higher adipogenic capacity than the mRNA-iPSC-MSC-YL001. However, both iPSCMSCs lines were derived from dermal fibroblasts. Thus, the adipogenic difference may be due to clonal variation, which has been reported in other studies $[42,43]$.

According to ClinicalTrail.gov to date, there are 452 studies registered for "mesenchymal stem cells", 32 studies registered for "induced pluripotent stem cells", and 27 studies registered for both "mesenchymal stem cells" AND "induced pluripotent stem cells". One key concern when using iPSCs and MSCs derived from iPSCs for cell therapy is their potential for teratoma formation. We have shown that the iPSC-MSCs generated by our method do not form teratoma after transplanting into immune deficient mice [16]. In this study, we further characterized a few "stemness" genes in the iPSC-MSCs. Except for KLF4 and C-MYC, the rest of the "stemness" genes (OCT4, SOX2, GDF3, CRIPTO, UTF1, DPPA4, DNMT3B, LIN28A, SALL4) were not expressed in the iPSC-MSC lines, further implicating the safety aspect of the iPSC-MSCs. KLF4 and C-MYC were also expressed in the BM-MSCs and in primary fibroblasts. Similarly, KLF4 and C-MYC were reported to be expressed in human fibroblasts by a previous study [8], suggesting that the presence of KLF4 and C-MYC should not contribute to teratoma formation by the iPSC-MSCs. With more studies conducted to characterize the safety and efficacy aspects of iPSC-MSCs, increasing numbers of clinical trials using iPSC-MSCs are expected in the future. We also observed an expression difference for KLF4 and C-MYC between the mRNA-iPSC-MSCYL001 and lenti-iPSC-MSC-A001 after osteogenic and adipogenic differentiation. Since $K L F 4$ and $C-M Y C$ are expressed in mRNA-iPSC-MSC-YL001 and lenti-iPSCMSC-A001 cells, the expression of KLF4 and C-MYC in osteogenic and adipogenic samples might have resulted from the proportion of parental iPSC-MSCs that failed to differentiate. The difference between the two iPSCMSC lines could have resulted from the different mesenchymal heterogeneity of these two iPSC-MSCs as shown in Fig. 2.

Another important challenge for generating clinically compliant iPSC-MSCs is the time-effective generation of a 
large amount of functional MSCs. Several methods have been described to derive functional MSCs from human iPSCs. These methods vary from each other in terms of ingtermediate embryoid bodies, surface coating of tissue culture vessels, differentiation medium, and supplement of pathway inhibitors (e.g., the p38 MAPK inhibitor, SB203580 and the transforming growth factor- $\beta$ pathway inhibitor SB431542) [32, 44]. The use of molecule SB431542 did not improve the adipogenic capacity of iPSC-MSCs [32]. However, robust adipogenesis of iPSC-MSCs has been achieved by supplementing the SB203580 inhibitor [44]. Our method for iPSC-MSC derivation involves neither intermediate embryoid bodies nor the use of pathway inhibitors. The derivation of iPSC-MSCs is achieved by MSC medium-mediated pre-differentiation, followed by MSC enrichment by serial passaging in MSC culture condition, which take approximately 1 month $[4,16]$. A similar method has been applied to derive functional MSCs by other groups $[42,45]$. Compared to the embryoid body-based differentiation method, this method is more time- and costeffective, and compliant with large-scale production. However, as proved in this study, further improvements are still required for our iPSC-MSC method to generate functional MSCs that can resemble the naïve BM-MSC functions.

\section{Conclusions}

The iPSC-MSCs derived by our method display most mesenchymal characteristics similar to adult BM-MSCs. The iPSC-MSCs achieve robust osteogenicity and chondrogenicity, but have very limited adipogenicity. Our results suggest that these iPSC-MSCs present a promising alternative cell source for MSC-based therapeutic applications.

\footnotetext{
Abbreviations

BM: Bone marrow; BM-MSC: Bone marrow-derived mesenchymal stem cell; BSA: Bovine serum albumin; DMEM: Dulbecco's modified Eagle's medium; ESC-MSC: Embryonic stem cell-derived mesenchymal stem cell; FBS: fetal bovine serum; iPSC: Induced pluripotent stem cell; iPSC-MSC: Induced pluripotent stem cell-derived mesenchymal stem cell; KSR: Knockout serum replacement; MSC: Mesenchymal stem cell; NHDF: Normal human dermal fibroblast; NPD: Number of population doublings; Ol: Osteogenesis imperfect; P: Passage; PBS: phosphate-buffered saline.
}

\section{Competing interests}

The authors declare that they have no competing interests.

\section{Authors' contributions}

RK, YZ and YL generated and characterized the lenti-iPSC and lenti-iPSC-MSC cell lines. RK, YZ and ST carried out the tri-lineage differentiation and characterization studies. YZ and ST performed the quantitative PCR. RK and $Y Z$ drafted the manuscript. RK and YL designed and conceived the study, and $G Z, L A, L X, C B$, and $L B$ participated in its design and coordination and helped to draft the manuscript. All authors read and approved the final manuscript.

\section{Acknowledgements}

This project is supported by grants from the Danish Research Council for Independent Research: Technology and Production Science (16942), the Sapere Aude Research Talent Prize (18382), the Lundbeck Foundation (DREAM) (R151-2013-14439), the Natural Science Foundation of China (81472126) to ST and GQZ, the Clinic and Natural Science Foundation of Jiangsu province of China (BL2012069, BK2012490), and the PhD scholarship from The China Scholarship Council (CSC) and the Faculty of HEALTH, Aarhus University to YZ. Flow cytometry was performed at the FACS Core Facility, Aarhus University, Denmark.

\section{Author details}

'Orthopedic Research Lab, Aarhus University, 8000 Aarhus C, Denmark. ${ }^{2}$ Jiangsu Province Hospital on Integration of Chinese and Western Medicine, Nanjing 210028, China. ${ }^{3}$ Department of Biomedicine, the Health Faculty, Aarhus University, 8000 Aarhus C, Denmark. ${ }^{4}$ Shenzhen Key Laboratory for Anti-aging and Regenerative Medicine, Health Science Center, Shenzhen University, 518060 Shenzhen, China.

Received: 13 February 2015 Revised: 23 February 2015

Accepted: 23 July 2015 Published online: 18 August 2015

\section{References}

1. Jiang $Y$, Jahagirdar BN, Reinhardt RL, Schwartz RE, Keene CD, Ortiz-Gonzalez $X R$, et al. Pluripotency of mesenchymal stem cells derived from adult marrow. Nature. 2002:418:41-9. doi:10.1038/nature00870.

2. Sasaki M, Abe R, Fujita $Y$, Ando $S$, Inokuma D, Shimizu H. Mesenchymal stem cells are recruited into wounded skin and contribute to wound repair by transdifferentiation into multiple skin cell type. J Immunol. 2008:180:2581-7.

3. Li WJ, Tuli R, Huang X, Laquerriere P, Tuan RS. Multilineage differentiation of human mesenchymal stem cells in a three-dimensional nanofibrous scaffold. Biomaterials. 2005;26:5158-66. doi:10.1016/j.biomaterials.2005.01.002.

4. Kang R, Luo Y, Zou L, Xie L, Lysdahl H, Jiang X, et al. Osteogenesis of human induced pluripotent stem cells derived mesenchymal stem cells on hydroxyapatite contained nanofibers. RSC Adv. 2014;4:5734-9. doi:10.1039/C3RA44181D.

5. Meinel L, Karageorgiou V, Fajardo R, Snyder B, Shinde-Patil V, Zichner L, et al. Bone tissue engineering using human mesenchymal stem cells: effects of scaffold material and medium flow. Ann Biomed Eng. 2004;32:112-22.

6. He Q, Wan C, Li G. Concise review: multipotent mesenchymal stromal cells in blood. Stem Cells. 2007;25:69-77. doi:10.1634/stemcells.2006-0335.

7. Bruder SP, Jaiswal N, Haynesworth SE. Growth kinetics, self-renewal, and the osteogenic potential of purified human mesenchymal stem cells during extensive subcultivation and following cryopreservation. J Cell Biochem. 1997;64:278-94.

8. Takahashi K, Tanabe K, Ohnuki M, Narita M, Ichisaka T, Tomoda K, et al. Induction of pluripotent stem cells from adult human fibroblasts by defined factors. Cell. 2007;131:861-72. doi:10.1016/..cell.2007.11.019.

9. Takahashi K, Yamanaka S. Induction of pluripotent stem cells from mouse embryonic and adult fibroblast cultures by defined factors. Cell. 2006;126:663-76. doi:10.1016/j.cell.2006.07.024.

10. Zhou H, Wu S, Joo JY, Zhu S, Han DW, Lin T, et al. Generation of induced pluripotent stem cells using recombinant proteins. Cell Stem Cell. 2009;4:381-4. doi:10.1016/j.stem.2009.04.005.

11. Warren L, Manos PD, Ahfeldt T, Loh YH, Li H, Lau F, et al. Highly efficient reprogramming to pluripotency and directed differentiation of human cells with synthetic modified mRNA. Cell Stem Cell. 2010;7:618-30.

12. Hou P, Li Y, Zhang X, Liu C, Guan J, Li H, et al. Pluripotent stem cells induced from mouse somatic cells by small-molecule compounds. Science. 2013;341:651-4. doi:10.1126/science.1239278.

13. Lian Q, Zhang Y, Zhang J, Zhang HK, Wu X, Lam FF, et al. Functional mesenchymal stem cells derived from human induced pluripotent stem cells attenuate limb ischemia in mice. Circulation. 2010;121:1113-23. doi:10.1161/CIRCULATIONAHA.109.898312.

14. Bilousova G, du Jun H, King KB, De Langhe S, Chick WS, Torchia EC, et al. Osteoblasts derived from induced pluripotent stem cells form calcified structures in scaffolds both in vitro and in vivo. Stem Cells. 2011;29:206-16. doi:10.1002/stem.566. 
15. Villa-Diaz LG, Brown SE, Liu Y, Ross AM, Lahann J, Parent JM, et al. Derivation of mesenchymal stem cells from human induced pluripotent stem cells cultured on synthetic substrates. Stem Cells. 2012;30:1174-81. doi:10.1002/stem.1084

16. Zou L, Luo Y, Chen M, Wang G, Ding M, Petersen CC, et al. A simple method for deriving functional MSCs and applied for osteogenesis in 3D scaffolds. Sci Rep. 2013;3:2243. doi:10.1038/srep02243.

17. Papapetrou EP, Sadelain M. Generation of transgene-free human induced pluripotent stem cells with an excisable single polycistronic vector. Nat Protoc. 2011;6:1251-73. doi:10.1038/nprot.2011.374.

18. Papapetrou EP, Lee G, Malani N, Setty M, Riviere I, Tirunagari LM, et al. Genomic safe harbors permit high beta-globin transgene expression in thalassemia induced pluripotent stem cells. Nat Biotechnol. 2011;29:73-8. doi:10.1038/nbt.1717

19. Stolzing A, Jones E, McGonagle D, Scutt A. Age-related changes in human bone marrow-derived mesenchymal stem cells: consequences for cell therapies. Mech Ageing Dev. 2008;129:163-73. doi:10.1016/j.mad.2007.12.002.

20. Nerurkar NL, Baker BM, Sen S, Wible EE, Elliott DM, Mauck RL. Nanofibrous biologic laminates replicate the form and function of the annulus fibrosus. Nat Mater. 2009;8:986-92. doi:10.1038/nmat2558.

21. Lysdahl H, Baatrup A, Nielsen AB, Foldager CB, Bunger C. Phenol red inhibits chondrogenic differentiation and affects osteogenic differentiation of human mesenchymal stem cells in vitro. Stem Cell Rev. 2013;9:132-9. doi:10.1007/s12015-012-9417-0.

22. LeGeros RZ, Craig RG. Strategies to affect bone remodeling: osteointegration. J Bone Miner Res. 1993;8:S583-96. doi:10.1002/jbmr.5650081328.

23. Quintanilla Jr RH, Asprer JS, Vaz C, Tanavde V, Lakshmipathy U. CD44 is a negative cell surface marker for pluripotent stem cell identification during human fibroblast reprogramming. PLoS One. 2014;9:e85419. doi:10.1371/journal.pone.0085419.

24. Dominici M, Le Blanc K, Mueller I, Slaper-Cortenbach I, Marini F, Krause D, et al. Minimal criteria for defining multipotent mesenchymal stromal cells. The International Society for Cellular Therapy position statement. Cytotherapy. 2006;8:315-7. doi:10.1080/14653240600855905.

25. Chung MT, Liu C, Hyun JS, Lo DD, Montoro DT, Hasegawa M, et al. CD90 (Thy-1)-positive selection enhances osteogenic capacity of human adipose-derived stromal cells. Tissue Eng Part A. 2013;19:989-97. doi:10.1089/ten.TEA.2012.0370.

26. Penick KJ, Solchaga LA, Welter JF. High-throughput aggregate culture system to assess the chondrogenic potential of mesenchymal stem cells. Biotechniques. 2005;39:687-91.

27. Maehr R, Chen S, Snitow M, Ludwig T, Yagasaki L, Goland R, et al. Generation of pluripotent stem cells from patients with type 1 diabetes. Proc Natl Acad Sci U S A. 2009;106:15768-73. doi:10.1073/pnas.0906894106.

28. Phillips MD, Kuznetsov SA, Cherman N, Park K, Chen KG, McClendon BN, et al. Directed differentiation of human induced pluripotent stem cells toward bone and cartilage: in vitro versus in vivo assays. Stem Cells Trans Med. 2014;3:867-78. doi:10.5966/sctm.2013-0154.

29. Giuliani M, Oudrhiri N, Noman ZM, Vernochet A, Chouaib S, Azzarone B, et al. Human mesenchymal stem cells derived from induced pluripotent stem cells down-regulate NK-cell cytolytic machinery. Blood. 2011;118:3254-62. doi:10.1182/blood-2010-12-325324

30. Jeong J, Shin K, Lee SB, Lee DR, Kwon H. Patient-tailored application for Duchene muscular dystrophy on mdx mice based induced mesenchymal stem cells. Exp Mol Pathol. 2014;97:253-8. doi:10.1016/j.yexmp.2014.08.001.

31. Lian Q, Zhang Y, Zhang J, Zhang HK, Wu X, Zhang Y, et al. Functional mesenchymal stem cells derived from human induced pluripotent stem cells attenuate limb ischemia in mice. Circulation. 2010;121:1113-23. doi:10.1161/CIRCULATIONAHA.109.898312.

32. Chen YS, Pelekanos RA, Ellis RL, Horne R, Wolvetang EJ, Fisk NM. Small molecule mesengenic induction of human induced pluripotent stem cells to generate mesenchymal stem/stromal cells. Stem Cells Trans Med. 2012;1:83-95. doi:10.5966/sctm.2011-0022.

33. Whitworth DJ, Frith JE, Frith TJ, Ovchinnikov DA, Cooper-White JJ, Wolvetang EJ. Derivation of mesenchymal stromal cells from canine induced pluripotent stem cells by inhibition of the TGFbeta/activin signaling pathway. Stem Cells Dev. 2014. doi:10.1089/scd.2013.0634.

34. Hoogduijn MJ, Crop MJ, Peeters AM, Van Osch GJ, Balk AH, ljzermans JN, et al. Human heart, spleen, and perirenal fat-derived mesenchymal stem cells have immunomodulatory capacities. Stem Cells Dev. 2007;16:597-604. doi:10.1089/scd.2006.0110.
35. Hu $Y$, Liao L, Wang $Q, M a ~ L, ~ M a ~ G$, Jiang $X$, et al. Isolation and identification of mesenchymal stem cells from human fetal pancreas. J Lab Clin Med. 2003;141:342-9. doi:10.1016/50022-2143(03)00022-2.

36. Kern S, Eichler H, Stoeve J, Kluter H, Bieback K. Comparative analysis of mesenchymal stem cells from bone marrow, umbilical cord blood, or adipose tissue. Stem Cells. 2006;24:1294-301. doi:10.1634/stemcells.2005-0342.

37. Deyle DR, Khan IF, Ren G, Wang PR, Kho J, Schwarze U, et al. Normal collagen and bone production by gene-targeted human osteogenesis imperfecta iPSCs. Mol Ther. 2012;20:204-13. doi:10.1038/mt.2011.209.

38. Koyama N, Miura M, Nakao K, Kondo E, Fujii T, Taura D, et al. Human induced pluripotent stem cells differentiated into chondrogenic lineage via generation of mesenchymal progenitor cells. Stem Cells Dev. 2013;22:102-13. doi:10.1089/scd.2012.0127.

39. Guzzo RM, Gibson J, Xu RH, Lee FY, Drissi H. Efficient differentiation of human iPSC-derived mesenchymal stem cells to chondroprogenitor cells. J Cell Biochem. 2013;114:480-90. doi:10.1002/jcb.24388.

40. Zhao Q, Gregory CA, Lee RH, Reger RL, Qin L, Hai B, et al. MSCs derived from iPSCS with a modified protocol are tumor-tropic but have much less potential to promote tumors than bone marrow MSCs. Proc Natl Acad Sci U S A. 2015;112:530-5. doi:10.1073/pnas.1423008112.

41. Boyd NL, Robbins KR, Dhara SK, West FD, Stice SL. Human embryonic stem cell-derived mesoderm-like epithelium transitions to mesenchymal progenitor cells. Tissue Eng Part A. 2009;15:1897-907. doi:10.1089/ ten.tea.2008.0351.

42. Hynes K, Menicanin D, Mrozik K, Gronthos S, Bartold PM. Generation of functional mesenchymal stem cells from different induced pluripotent stem cell lines. Stem Cells Dev. 2014;23:1084-96. doi:10.1089/scd.2013.0111.

43. Kuci Z, Seiberth J, Latifi-Pupovci H, Wehner S, Stein S, Grez M, et al. Clonal analysis of multipotent stromal cells derived from CD271+ bone marrow mononuclear cells: functional heterogeneity and different mechanisms of allosuppression. Haematologica. 2013;98:1609-16. doi:10.3324/ haematol.2013.092700

44. Wei H, Tan G, Manasi, Qiu S, Kong G, Yong P, et al. One-step derivation of cardiomyocytes and mesenchymal stem cells from human pluripotent stem cells. Stem Cell Res. 2012;9:87-100. doi:10.1016/j.scr.2012.04.003.

45. Hynes K, Menicanin D, Gronthos S, Bartold MP. Differentiation of iPSC to mesenchymal stem-like cells and their characterization. Methods Mol Biol. 2014. doi:10.1007/7651_2014_142.

\section{Submit your next manuscript to BioMed Central and take full advantage of:}

- Convenient online submission

- Thorough peer review

- No space constraints or color figure charges

- Immediate publication on acceptance

- Inclusion in PubMed, CAS, Scopus and Google Scholar

- Research which is freely available for redistribution

Submit your manuscript at www.biomedcentral.com/submit
C) Biomed Central 